Check for updates

Received 23rd July 2018

DOI: $10.1039 / c 8 r a 05982 a$

rsc.li/rsc-advances
Cite this: RSC Adv., 2018, 8, 33347

Accepted 9th September 2018

\section{The effect of shear on the cytoskeleton remodeling and physiological performance of myocardium cells through Tmod1}

\begin{abstract}
Liang Zhao, (D) *abc Xiafei Li, ${ }^{b}$ Pei Niü ac and Li Lic
Objective: mechanical stimulation alters cell metabolism, but little is known about the effects of mechanical strain on the cytoskeleton of myocardium cells. This study was to investigate the changes of F-actin, a cytoskeleton protein of myocardium cells, and to provide a theoretical basis for further investigation of the mechanism of myocardium-remodeling. Methods: we examined the effects of fluid shear stress on the Tmod1 expression and F-actin cytoskeleton remodeling. Then, after myocardial cells, silenced by siTmod1, were treated by fluid shear stress, the change of intracellular calcium ion concentration, ROS in myocardial cells, cytochrome $\mathrm{C}$, and the amount of F-actin, LDH and T-SOD MDA were evaluated with laser light confocal microscopy, western blot, and ELISA, respectively. Results: fluid shear stress can induce F-actin cytoskeleton remodeling and upregulate Tmod1 expression. After myocardial cells were under the conditions of Tmod1 inhibition, shear stress can significantly reduce the increase of ROS levels and calcium content, decrease the release of cells cytochrome $C$ and $L D H$, decrease the MDA content, and increase the level of T-SOD. Conclusion: in conclusion, shear treatment can remodel the cytoskeleton through Tmod1, and its mechanism may be related to scavenging oxidative stress products, ROS and MDA, the increase of intracellular antioxidant enzyme activity of SOD and improvement in mitochondrial dysfunction.
\end{abstract}

\section{Introduction}

The cytoskeleton is an important structure to maintain the basic morphology of cells. ${ }^{1}$ The cytoskeleton is composed of microfilaments, microtubules and intermediate fibers. Microfilaments are ubiquitous in a variety of cells and crosslink with a variety of membrane proteins. ${ }^{2}$ It has a critical role in the movement, maintenance and division of cell morphology. Microfilaments are made up of actin and myosin, and actin is a basic protein that can reflect the morphological changes of microfilaments. The regulatory effects of various regulatory proteins on actin and myosin are necessary for the shape change of cells and the contraction of myocardium. Tropomodulin (Tmod) are proteins that bind to tropomyosin in the cytoskeleton. After binding, the actin itself and its actin were also blocked. ${ }^{3}$

Tropomodulin family possesses four mammalian Tmods (Tmods 1-4) that are about are $70 \%$ similar in amino acid sequence. They are expressed in different tissues with

${ }^{a}$ PKU-HKUST Shenzhen-Hongkong Institution, Shenzhen, China. E-mail: zhaoliang4321@163.com

${ }^{b}$ Institute of Life Science and Health, College of Life Sciences and Technology, Xinxiang Medical University, Xinxiang, Henan, China

${ }^{c}$ Department of Mechanics and Engineering Science, College of Engineering, Peking University, Beijing, China developmentally regulated fashion. Tmod1 was also named as E-Tmod, ${ }^{4}$ which is mainly expressed in terminally differentiated cells (Fig. 1). Tmod1 interacts with the thin filament components actin and tropomyosin. All the Tmod proteins have a tropomyosin/Pointed-End Tropomyosin Capping (TM-Cap) domain at their N-terminal segment, followed with a LeucineRich Repeat/Pointed-End Actin Capping (LRR-Cap) domain. ${ }^{5}$ The functional regions within Tmod1 have tropomyosinbinding regions and actin-binding regions. So for the cell filament regulation, Tmod1 is very important. For example, studies in primary cultures of cardiomyocytes showed that either decreased expression of Tmod1 resulted in thin filament elongation or shortening, respectively. ${ }^{6}$

During the development and maturation of cells, cells come into being membrane skeleton. In the myocardial cells differentiation, the gene expressions of some membrane skeleton proteins are vitally increased. Many factors, such as chemical factors, biomechanical factors, can affect the myocardial cells formation. Mechanical factors could affect cell migration, proliferation, apoptosis and differentiation, as well as tissue development. So mechanical stimulation like fluid shear stress have important role in regulating of expression of cytoskeleton related genes.

In this article, we mainly study the role of mechanical factors in cytoskeletal remodeling, especially for Tmod1 expression. Then after myocardial cells silenced by si-Tmod1 was treated 


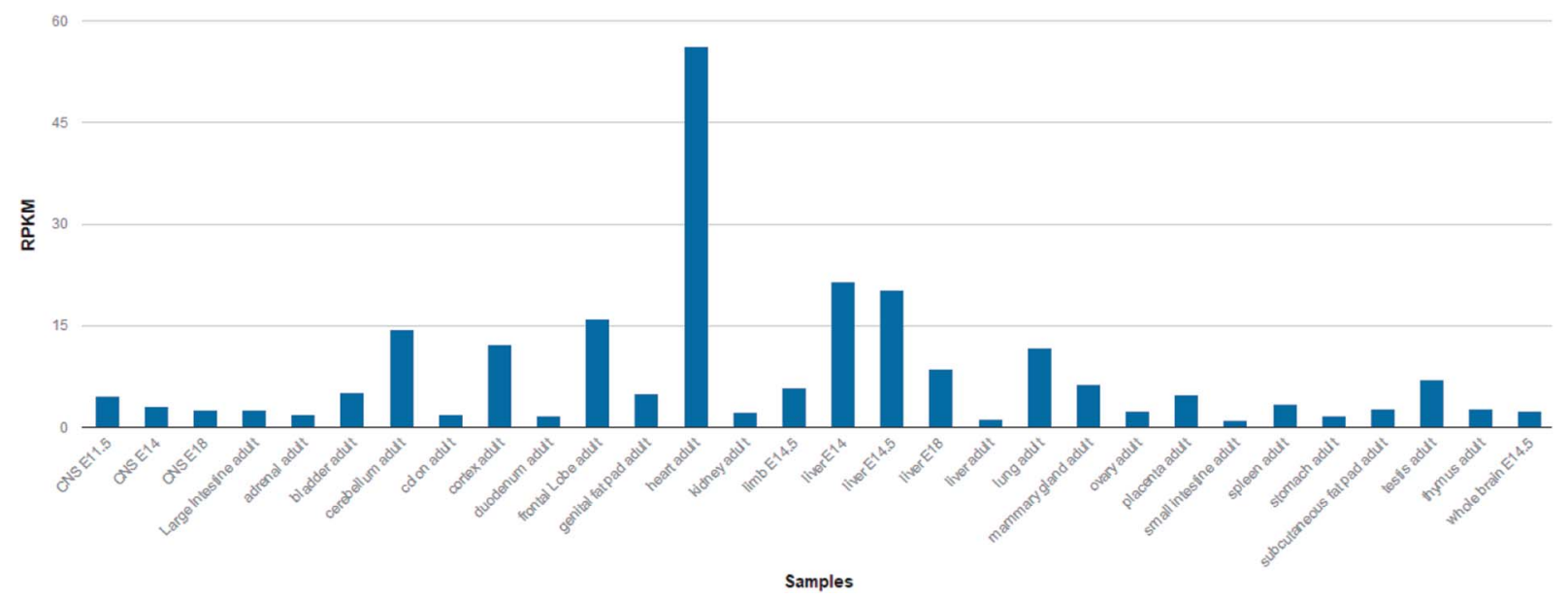

Fig. 1 The expression level of Tmod1 protein in other tissues is very low, however the expression level in the heart tissue is high. (from NCBI gene).

with fluid shear stress, the change of intracellular calcium ion concentration, ROS in myocardial cells, cytochrome C, the amount of F-actin, LDH, super oxide dismutase (SOD) and malondialdehyde (MDA) were evaluated with laser light confocal microscopy, western blot, ELISA, respectively.

\section{Materials and methods}

\subsection{Cell exposure to shear stress}

After the myocardial cells were cultured on the cover glass surface with DMEM medium containing $10 \%$ FBS for $24 \mathrm{~h}$, they were given $225 \times g$ relative centrifugal force for $10 \mathrm{~min}$ in each hour with the centrifuge in experimental group.

\subsection{Effect of shear stress on cytoskeleton remodeling}

After the myocardial cells was treated by fluid shear stress for $16 \mathrm{~h}$, fluorescein isothiocyanate (FITC) phalloidin was used to stain the cells according to the instruction. Then after being washed with PBS for three times, the myocardial cells were counterstained with 4',6-diamidino-2-phenylindole (DAPI, Beyotime, China) for $5 \mathrm{~min}$ and then were cleaned with PBS for three times. The cells were suspended in a mixture of glycerol and PBS (v/v, $1: 1)$ and placed in a confocal dish. The samples were observed by Nikon A1 laser scanning confocal microscope (Nikon instruments Co., Ltd, Japan). Image J software was used to calculate the average fluorescent intensity of the images.

\subsection{Tmod1 change in myocardial cells by fluid shear stress}

After the myocardial cells was treated by fluid shear stress for $8 \mathrm{~h}$ or $16 \mathrm{~h}$, TRIeasyTM Total RNA Extraction Reagent was used to extract the full RNA following the instructions of manufacturer. All the RNA was reverse transcribed to cDNA with a HieffTM First Strand cDNA Synthesis Super Mix for RT-qPCR (+gDNA wiper) (Shanghai yesen biological technology Co., Ltd, China). Real-time PCR was performed with a lightCycler ${ }^{\circledR} 96$ Quantitative PCR instrument with HieffTM qPCR SYBR ${ }^{\circledR}$ Green Master Mix (Shanghai Yesen Biological Technology Co., Ltd,
China). The primer sequences used for Tmod1 were as follows: forward primer: 5'-AATCCAATCGGGTCACAGGAG-3'; reverse primer: $5^{\prime}$-CTCGCACACCGACAGGTAG-3'. GAPDH (forward primer: $5^{\prime}$-ACCACAGTCCATGCCATCAC-3'; reverse primer: $5^{\prime}$ TCCCCACCCTGTTGCTGTA-3') was used in internal control group. A relative change in gene expression was calculated by the method of $2^{-\Delta \Delta C_{\mathrm{T}}}$.

\subsection{Determination of ROS generation and calcium content}

Reactive oxygen species (ROS) detection was used ROS Assay Kit (Shanghai yesen biological technology co., LTD, China). The oxidative conversion of $2^{\prime}, 7^{\prime}$-dichlorofluorescein diacetate (DCFH-DA) to fluorescent dichlorofluorescein (DCF) was used to measure the intracellular ROS level change with fluorescent spectrophotometer. Cells in confocal Petri dish were incubated with DMEM media. The samples were cleaned with PBS, and then incubated by DCFH-DA at $37^{\circ} \mathrm{C}$ for $20 \mathrm{~min}$. Nikon A1 laser scanning confocal microscope was used to observe the DCF fluorescence at an excitation wavelength of $488 \mathrm{~nm}$ and at an emission wavelength of $535 \mathrm{~nm}$. Calcium content was detected with Fluo-4, AM(Merck co., LTD, Germany) as the similar procedure as DCFH-DA.

\subsection{Measurement of cytochrome C protein expression by western blot}

Cells with $6 \times 10^{5}$ per bottle was inoculated in $50 \mathrm{~mL}$ culture bottle, and after culture for $24 \mathrm{~h}$, SDS total protein extraction kit (Beyotime, China) was used to extract protein. The protein concentration in each sample extract was determined according to the Bradford method using the BCA assay reagent (Beyotime, China), and all samples was then adjusted to the same value using $5 \times$ SDS-PAGE sample buffer. The samples were boiled for $5 \mathrm{~min}$, and then loaded in a 10\% SDS-PAGE gel (50 $\mu \mathrm{g}$ protein $/ 10$ $\mu \mathrm{L}$ per well) for electrophoresis with a WEALTEC gel apparatus at $100 \mathrm{~V}$ for $2 \mathrm{~h}$. The protein on the SDS-PAGE gel was transferred to the PVDF membrane and electrophoresed at $60 \mathrm{~V}$ for $2 \mathrm{~h}$. First, cytochrome C antibody (Catalog number: 10993-1-AP, 
Proteintech Group, Inc, USA) and secondary antibody (goat polyclonal secondary antibody to rabbit IgG-HRP, 1:500, Beyotime, China) were probed with PVDF membrane, and then treated by ECL Plus western blotting detection reagent at room temperature for $5 \mathrm{~min}$. The bands on the membrane were visualized with FUSION FX gel imaging analysis system and analyzed by image J software. GAPDH was used to normalize final reported data.

\subsection{Measurement of LDH, MDA, T-SOD, and Tmod1 expression levels with ELISA}

Levels of LDH (Catalog number: bsk00581, Bioss, China), MDA (Catalog number: bsk00545, Bioss, China), Tmod1 (Catalog number: PRA5764, Abebio, China) and T-SOD (Catalog number: S0101, Beyotime, China) were determinated by commercial ELISA kits. Each assay was conducted according to manufacturer's instructions. Standards at a series of concentrations were also detected. The concentrations of the samples were calculated according to the corresponding standard curve.

\section{Results}

\subsection{Cytoskeleton remodeling}

As morphological changes are associated with cytoskeleton remodeling, the F-actin content in cardiomyocytes under shear stress was detected. Confocal result showed that the F-actin content in cardiomyocytes increased 1.6 times by shear stress (Fig. 2).

\subsection{Fluid shear stress upregulates Tmod1 in myocardial cells}

As the terminated protein of F-actin, Tmod1 is the key to Factin stability and polymerization. ${ }^{7}$ It can play a part in $\mathrm{F}$ actin cytoskeleton remodeling induced by fluid shear stress. Therefore, we studied the expression of Tmod1 in cardiomyocytes treated by shear stress. Quantitative RT-PCR result showed that the expression of Tmod1 mRNA was increased by $8 \mathrm{~h}$ of shear (Fig. 3A). With the prolongation of shear time, the level of Tmod1 mRNA increased (Fig. 3B). The data indicate that fluid shear stress can increase the expression of Tmod1. Elisa experiment also got the similar result (Fig. 3C).

\subsection{The effect of Tmod1 knockdown on the content of F- actin in myocardial cells}

Tmod1 expression play an important in F-actin cytoskeleton remodeling. Therefore, we carried out a functional loss experiment to study the role of Tmod1 in the remodeling of Factin. When Tmod1 was knocked down with Tmod1-specific siRNA (Fig. 4A and B), F-actin expression amount was significantly decreased (Fig. 4C and D). These experiment results displayed that Tmod1 affected the content of F-actin in cardiac myocytes, which may mediate shear stress induced F-actin cytoskeleton remodeling.

\subsection{The influence of shear on the ROS, MDA, T-SOD and content of calcium ion within myocardial cells under the condition of Tmod1 inhibition}

ROS, MDA and T-SOD are important indexes for detecting oxidative damage and antioxidative damage in cells. ${ }^{8}$ Compared with control group, ROS fluorescence intensity and mitochondrial calcium fluorescence intensity significantly enhanced in si-Tmod1 group. Compared with si -Tmod1 group, shear group can significantly reduce the increasement of ROS levels and calcium content produced by Tmod1 restrain $(P<0.05)$. Compared with the control group, the amount of MDA expression raised evidently, while the content of T-SOD significantly decreased in si-Tmod1 group $(P<0.05)$. Compared with siTmod1 group, shear treatment could significantly decrease the MDA content, and increase the level of T-SOD $(P<0.05)$ (Fig. 5).

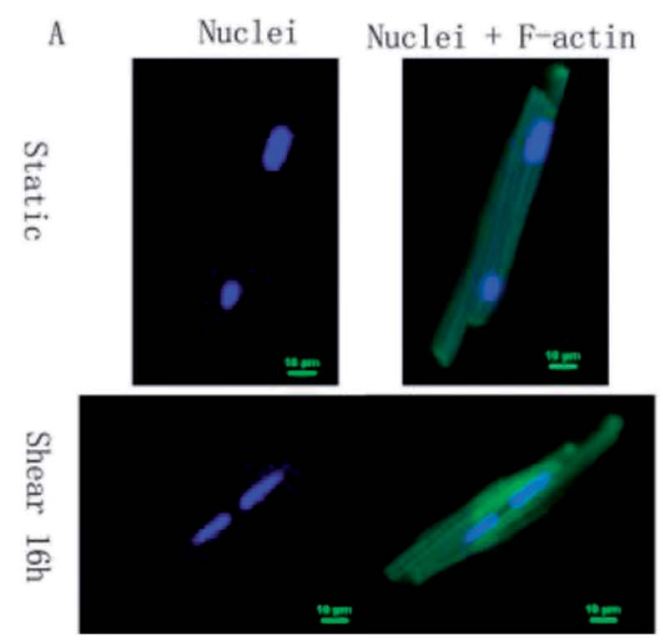

B

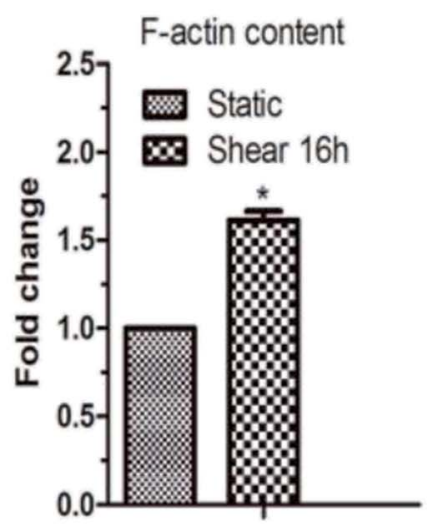

Fig. 2 F-actin cytoskeleton remodeling observed by laser scanning confocal microscope was induced by shear stress in cardiac myocytes (A), and the F-actin content change was manifested in (B). Compared with static group, *: $P<0.05, n=6$. 

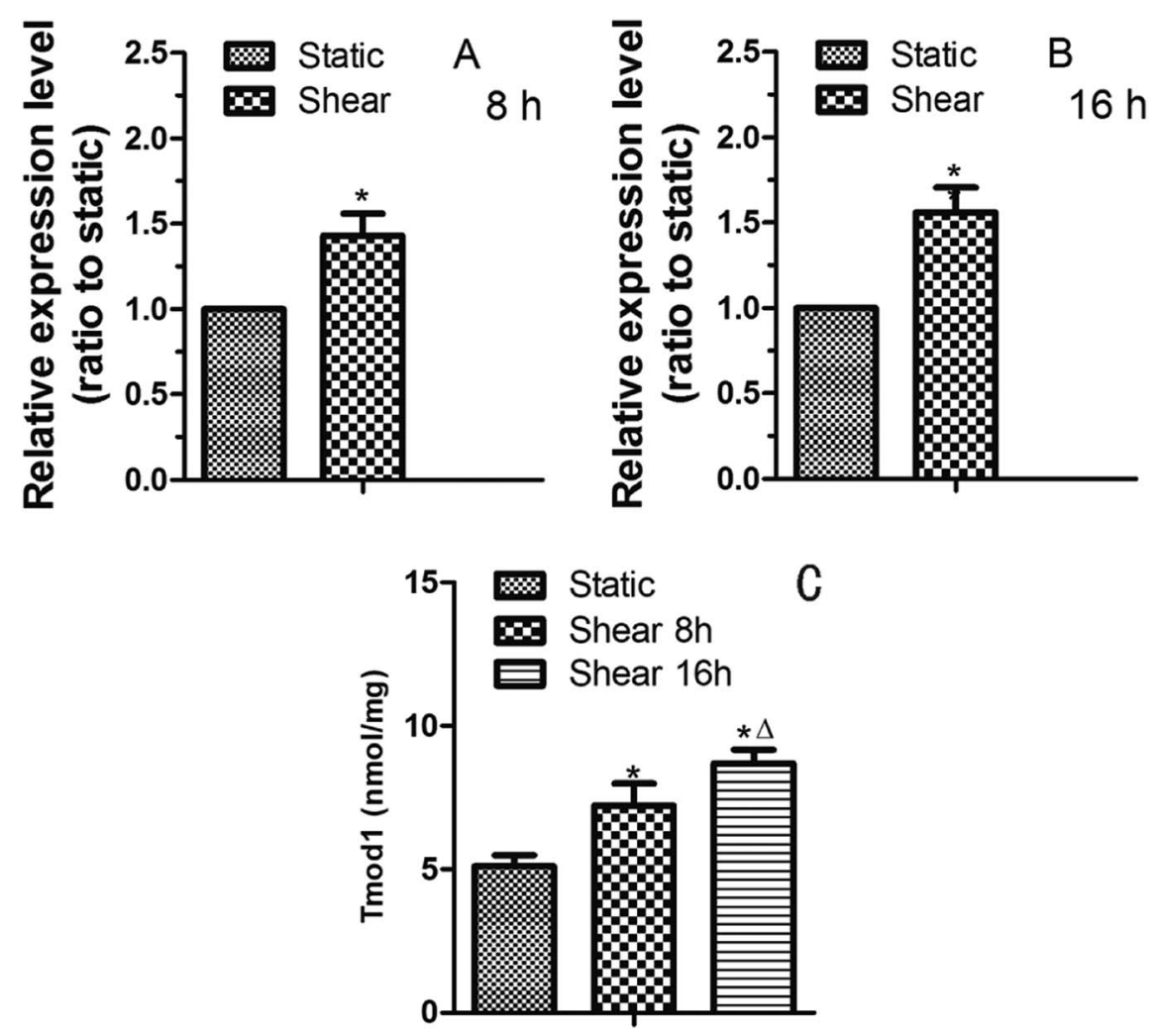

Fig. 3 Tmod1 mRNA expression levels were upregulated in myocardial cells induced by fluid shear stress for $8 \mathrm{~h}(\mathrm{~A}), 16 \mathrm{~h}$ (B), as detected with RTPCR. (C) Elisa experiment result indicated that Tmod1 expression was significantly up-regulated. *: $P<0.05$, compared to static group, ${ }^{\Delta}: P<0.05$, compared to shear $8 \mathrm{~h}$ group $n=6$.

\subsection{The effect of shear on myocardial cells cytochrome $\mathrm{C}$ release and $\mathrm{LDH}$}

Cytochrome $\mathrm{C}$ is released and initiator caspase- 9 activation may occur through cytosolic procaspase-9, which suggests that this is an important event in apoptosis. ${ }^{9} \mathrm{LDH}$ is an important enzyme in the process of energy metabolism in the body. This enzyme is commonly used in the diagnosis of myocardial infarction, liver disease and some malignant tumors. ${ }^{10}$ Compared with control group, the release of cytochrome $\mathrm{C}$ in the cell was significantly increased in si-Tmod1 group $(P<0.05)$. However, shear processing can obviously decrease the release of cells cytochrome $\mathrm{C}(P<0.05)$. The results are shown in Fig. 6 .

Compared with the control group, the LDH release amount in si-Tmod1 group of group was significantly higher $(P<0.05)$. Shear treatment can significantly reduce the release of LDH in the supernatant of cells, which suggested that shear may have a protective effect on myocardial cells treated with si-Tmod1 (Fig. 6).

\section{Discussion}

The physical activities of cardiac muscle cells and tissues are mainly manifested in two aspects: mechanical and electrical changes. The appropriate mechanical environment is a positive stimulus to the growth of myocardial cells, and can overcome the shortcomings of the traditional myocardial culture to a certain extent. Mechanical factors play an important role in the differentiation and growth of cardiomyocytes. Fluid shear stress has some influence on the formation of cardiovascular system. It has been reported that fluid shear stress can induce mesenchymal stem cells to differentiate into cardiovascular tissue cells. Xi et al. $^{\mathbf{1 1}}$ exerted pressure and shear force on the ventricular myocytes of $\mathrm{SD}$ rats by centrifugal method. By centrifugation $12 \mathrm{~h}$ or $24 \mathrm{~h}$, according to the flow cytometry and lactate dehydrogenase assay, $180 \mathrm{rpm}$ centrifugation affect the cell number and activity for the minimum, which is most suitable for cell culture.

Laser confocal microscopy analysis indicated that F-actin cytoskeleton content in cardiomyocytes was increased because of the fluid shear stress compared with control group (Fig. 2). This indicated that mechanical stimulation leaded to the remodeling of F-actin cytoskeleton, and prompts cardiomyocytes to resist to mechanical damage and cells show the mechanical adaptability. In this study, it was found that fluid shear stress can increase Tmod1 expression (Fig. 3). Tmod1 knockdown experiment indicated that Tmod1 affected F-actin content (Fig. 4), which can explain to some extent the molecular mechanism of mechanical stimulation that leads to the structural remodeling of cytoskeleton.

Mitochondria in myocardial cells play an important role in physiological function, and mitochondrial dysfunction can lead to ROS production increment in the mitochondria, and so increase mitochondrial calcium overload. ${ }^{\mathbf{1 2}}$ Physiological doses 
A
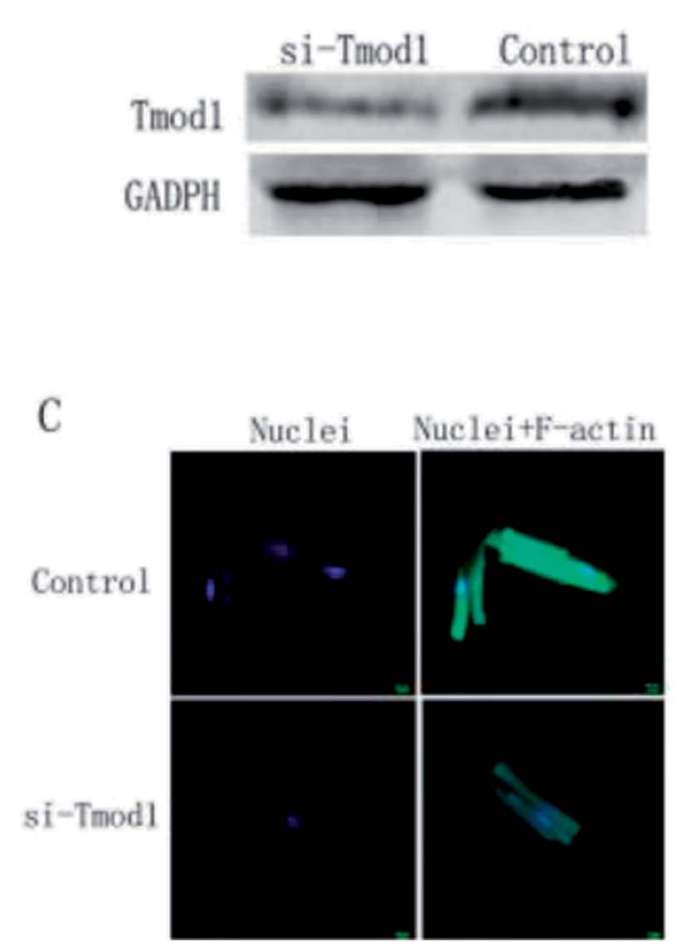

B
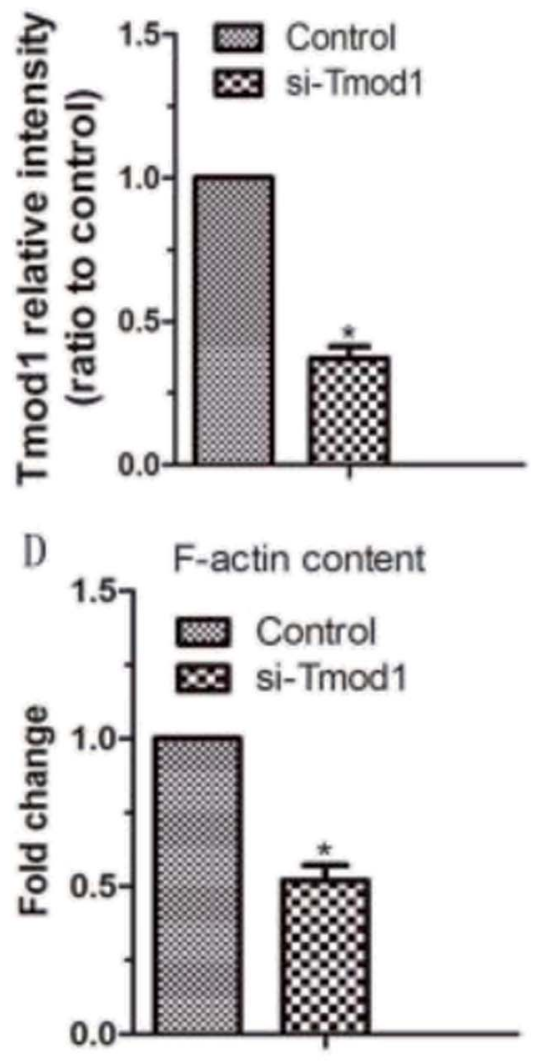

Fig. 4 siTmod1 inhibited F-actin expression in myocardial cells. (A and B) Tmod1 protein expression level was decreased in myocardial cells transfected with si-Tmod1. (C) F-actin fluorescence intensity stained with FITC phalloidin was decreased in myocardial cells transfected with $100 \mathrm{nM}$ si-Tmod1. The fold changes in F-actin expression amount were displayed in (D). *: $P<0.05$, compared to static group, $n=6$.

of ROS occupy the important position in mitochondrial enzyme system. The overproduction of ROS, will trigger oxidative stress and increase myocardial cell apoptosis and necrosis. ${ }^{13}$ This experiment found that shear processing on heart muscle cells, can significantly decrease the mitochondrial production of ROS and MDA content caused by si-Tmod1 processing, and improve the vigor of antioxidant kinase SOD. So the reason for shear resistance to oxidative stress resulted by si-Tmod1 processing is that it can balance of oxidative stress product, such as MDA and ROS, and the SOD enzyme in antioxidant stress system.

Mitochondria produces large amounts of ROS which exerts a role in the body of calcium overload, and excessive ROS calcium ion cause calcium balance disorder and mitochondrial dysfunction. ${ }^{\mathbf{1 4 1 5}}$ A large number of ROS and mitochondrial calcium overload accelerated the opening of mitochondrial permeability transition pore ${ }^{\mathbf{1 6}}$ and further lead to the disorder of mitochondrial membrane potential, resulting in a large number of cytochrome $\mathrm{C}$ release, triggering the formation of the pro apoptotic caspase family, which caused apoptosis and necrosis of cells. We found that shear treatment could significantly reduce mitochondrial calcium overload caused by siTmod1 treatment and inhibit the release of cytochrome C. It suggests that oxidative stress induced by shear treatment against si-Tmod1 may be related to the improvement of mitochondrial dysfunction.

$\mathrm{LDH}$ is an important index for detecting the degree of myocardial injury. LDH mainly exists in human tissues, belongs to hydrogen transfer enzymes, catalyzing the oxidation of lactic acid to pyruvate. ${ }^{17,18}$ As long as there is a small amount of tissue necrosis in human tissues, the enzyme can increase obviously. In routine physical examination, biochemical LDH examination has been used as a routine physical examination item. ${ }^{19}$ Wang et al. transfected H9C2 cardiomyoblasts with lentivirus expressing miR-214 (LmiR-214) or lentivirus expressing scrambled miR-control (LmiR-control) respectively. The cells were subjected to hypoxia for $4 \mathrm{~h}$ followed by reoxygenation for $24 \mathrm{~h}$. Transfection of LmiR-214 suppresses PTEN expression, significantly increases the levels of Akt phosphorylation, markedly attenuates $\mathrm{LDH}$ release. The in vitro and in vivo data suggests that miR-214 protects cells from $\mathrm{H} / \mathrm{R}$ induced damage and attenuates I/R induced myocardial injury. ${ }^{20}$ In this paper, shear treatment obviously decrease the LDH content, which has a significant difference in statistics with si-Tmod1 group.

In conclusion, shear treatment has a protective effect on myocardial cells treated with si-Tmod1, and its mechanism may be related to decrease of oxidative stress products MDA and 

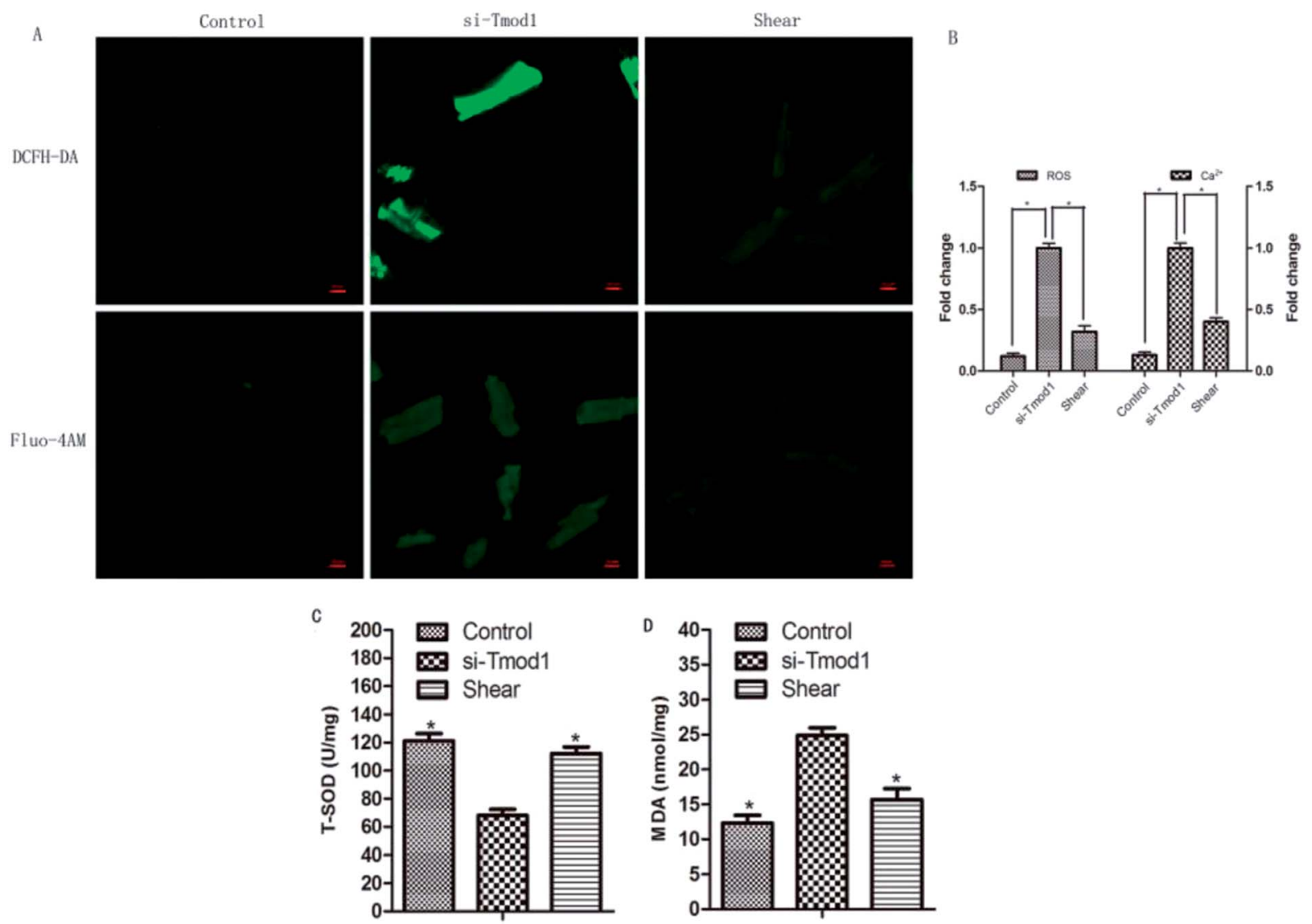

Fig. 5 Effect of fluid shear on the expression of mitochondrial ROS, MDA, T-SOD and mitochondrial calcium in myocardial cells by knockdown of Tmod1. (A) The production of mitochondrial ROS (DCFH-DA green fluorescent image) and mitochondrial calcium (Fluo-4AM green fluorescent) were estimated by confocal microscopy. (B) Quantification of the production of mitochondrial ROS and the level of mitochondrial calcium by analysis software of confocal microscopy. (C) Effect of fluid shear decrease the MDA content in cardiomyocyte undergoing si-Tmod1 treatment. (D) Effect of fluid shear increase the T-SOD content in cardiomyocyte undergoing si-Tmod1 treatment. $(* P<0.05$, other groups vs. siTmod1 group, $n=6$ ).

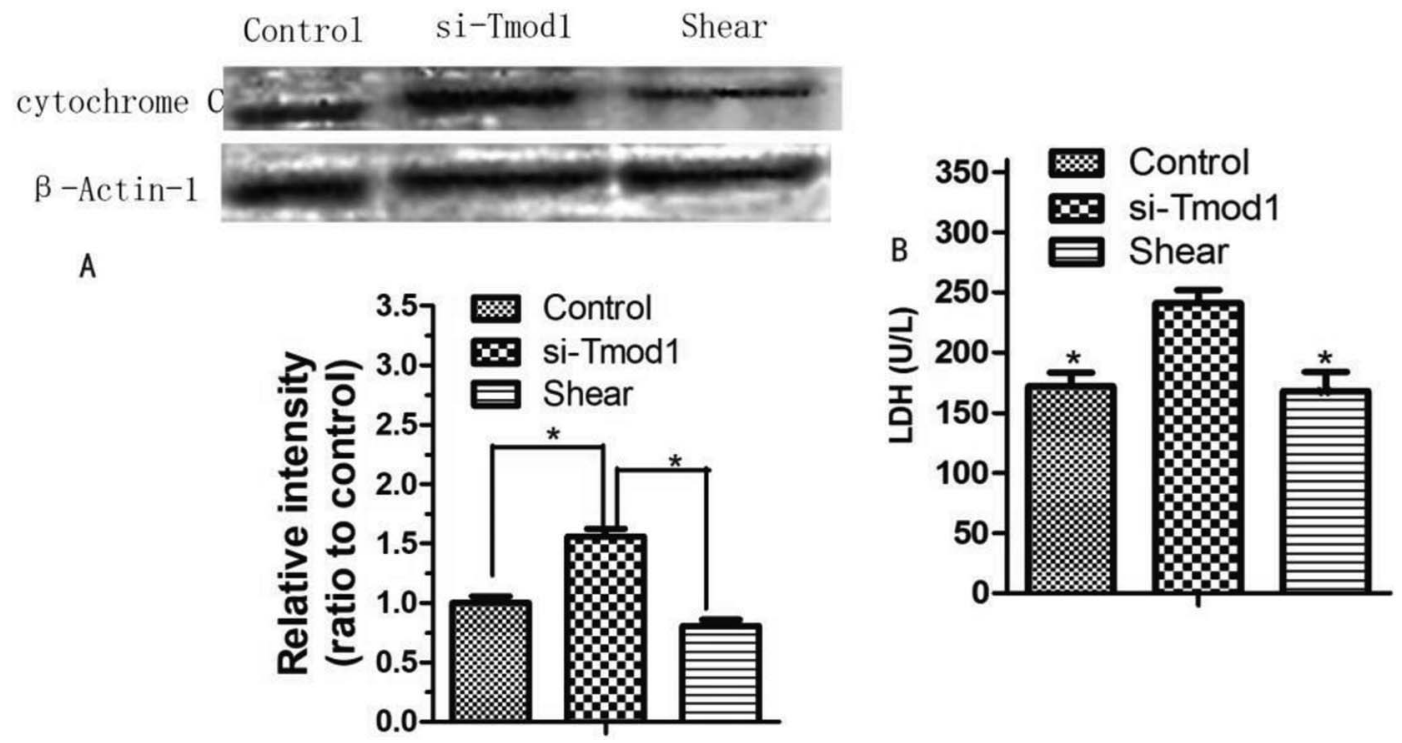

Fig. 6 (A) Effect of fluid shear on cytochrome-c release in myocardial cells suffering by knockdown of Tmod1. (B) Effect of fluid shear decrease the LDH content in cardiomyocyte undergoing si-Tmod1 treatment. ( $* P<0.05$, other groups vs. si-Tmod1 group, $n=6$ ). 
ROS, increase of intracellular antioxidant enzyme activity of SOD and mitochondrial dysfunction improvement.

\section{Sources of funding}

This research is supported in part by Shenzhen Science and Technology R\&D Grant JCYJ20160427170536358, Scientific Research Fund of Xinxiang Medical University 2014QN138; and Key Scientific Research Project of Henan Institutions of Higher Learning 17A180034.

\section{Conflicts of interest}

The authors declared that they have no conflicts of interest to this work. We declare that we do not have any commercial or associative interest that represents a conflict of interest in connection with the work submitted.

\section{Acknowledgements}

We thank all participants of the study in College of Engineering, Peking University and College of Medicine, Hebei University.

\section{References}

1 P. K. Mattila, F. D. Batista and B. Treanor, J. Cell Biol., 2016, 212, 267-280.

2 F. W. Wang, F. Zhao, X. Y. Qian, Z. Z. Yu, J. Zhao, L. Su, Y. Zhang, S. L. Zhang, B. X. Zhao and J. Y. Miao, RSC Adv., 2014, 4, 56722-56730.

3 V. M. Fowler, Curr. Top. Membr., 2013, 72, 39-88.

4 S. Yamashiro, D. S. Gokhin, S. Kimura, R. B. Nowak and V. M. Fowler, Cytoskeleton, 2012, 69, 337-370.

5 T. Suzuki, A. Kasamatsu, I. Miyamoto, T. Saito, M. Higo, Y. Endosakamoto, M. Shiiba, H. Tanzawa and K. Uzawa, Int. J. Oncol., 2016, 48, 607.
6 D. S. Gokhin, J. Ochala, A. A. Domenighetti and V. M. Fowler, Development, 2016, 142, 4351-4362.

7 C. Cheng, R. B. Nowak, S. K. Biswas, W. K. Lo, P. G. Fitzgerald and V. M. Fowler, Invest. Ophthalmol. Visual Sci., 2016, 57, 4084.

8 W. Li, S. Zhao, F. Cheng, T. Rao, W. Yu, Y. Ruan, R. Yuan and X. Yao, Mol. Med. Rep., 2018, 17, 6819-6827.

9 C. M. Carthy, B. Yanagawa, H. Luo, D. J. Granville, D. Yang, P. Cheung, C. Cheung, M. Esfandiarei, C. M. Rudin, C. B. Thompson, D. W. C. Hunt and B. M. McManus, Virology, 2003, 313, 147-157.

10 T. Dong, Z. Liu, Q. Xuan, Z. Wang, W. Ma and Q. Zhang, Sci. Rep., 2017, 7, 6069.

11 Y. Xi, G. Wu, X. Bai, T. Geng and A. Ma, Eur. J. Heart Failure, 2006, 8, 16-22.

12 G. Chang, D. Zhang, J. Liu, P. Zhang, L. Ye, K. Lu, Q. Duan, A. Zheng and S. Qin, Exp. Biol. Med., 2014, 239, 414-422.

13 Q. Li, D. Su, B. O'Rourke, S. M. Pogwizd and L. Zhou, Am. J. Physiol.: Heart Circ. Physiol., 2015, 308, H623.

14 S. Jang and S. Javadov, Arch. Biochem. Biophys., 2017, 630, 1. 15 F. Scialò, D. J. Fernándezayala and A. Sanz, Front. Physiol., 2017, 8, 428.

16 K. Seo, S. Seo, S. H. Ki and S. M. Shin, Biol. Pharm. Bull., 2016, 39, 799-806.

17 S. J. Allison, J. R. P. Knight, C. Granchi, R. Rani, F. Minutolo, J. Milner and R. M. Phillips, Oncogenesis, 2014, 3, e102.

18 M. Gallo, L. Sapio, A. Spina, D. Naviglio, A. Calogero and S. Naviglio, Front. Biosci., 2015, 20, 1234.

19 P. Miao, S. Sheng, X. Sun, J. Liu and G. Huang, IUBMB Life, 2013, 65, 904-910.

20 X. Wang, T. Ha, Y. Hu, C. Lu, L. Liu, X. Zhang, R. Kao, J. Kalbfleisch, D. Williams and C. Li, Oncotarget, 2016, 7, 86926-86936. 